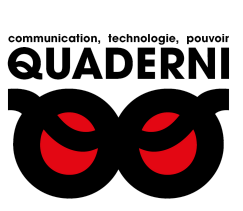

\title{
Quaderni
}

Communication, technologies, pouvoir

96 | Printemps 2018

Smart city : "fiction" et innovation stratégique

\section{La smart city pour voir et concevoir autrement la ville contemporaine}

Jean-Marc Offner

\section{(2) OpenEdition \\ 12 Journals}

\section{Édition électronique}

URL : https://journals.openedition.org/quaderni/1172

DOI : 10.4000/quaderni.1172

ISSN : 2105-2956

Éditeur

Les éditions de la Maison des sciences de l'Homme

Édition imprimée

Date de publication : 15 mai 2018

Pagination : 17-27

Référence électronique

Jean-Marc Offner, "La smart city pour voir et concevoir autrement la ville contemporaine », Quaderni

[En ligne], 96 | Printemps 2018, mis en ligne le 15 mai 2020, consulté le 28 septembre 2021. URL

http://journals.openedition.org/quaderni/1172 ; DOI : https://doi.org/10.4000/quaderni.1172 


\section{$D$ ossier}

\section{La smart city}

pour voir et concevoir autrement la ville

\section{contemporaine}

\section{Jean-Marc Offner}

Directeur général de l'a-urba (agence d'urbanisme Bordeaux métropole Aquitaine) Président du conseil stratégique de l'École urbaine de Sciences Po Ancien directeur du Latts (Laboratoire TechniquesTerritoires-Sociétés)
Que dire d'intelligent sur la smart city qui n'ait pas déjà été écrit et répété ? Depuis le début de la construction du quartier « hyperconnecté » de Songdo en Corée du Sud, au début des années 2000, depuis l'appel de Bill Clinton au géant informatique Cisco en 2005 pour décongestionner les métropoles, la ville intelligente commence à remplacer la ville durable dans la rhétorique urbaine des techniciens et des politiques. L'Union européenne a amplifié le processus, mettant le sujet à son agenda urbain. La scène publique semble devoir ainsi recycler de plus en plus rapidement ses "mythes opératoires ", faute peut-être d'une inventivité locale mobilisatrice.

La ville durable voulait et veut sauver la planète... Cette ambition peu contestable a longtemps fait taire les critiques. Plus ambiguë dans ses objectifs, la ville intelligente a plus rapidement suscité les méfiances, entre suspicion de surveillance généralisée par les pouvoirs publics et mainmise des capitaux privés sur l'administration des villes. Aussi un contrediscours s'est-il vite installé : Participolis plutôt que Datapolis (pour reprendre les termes de Francis Pisani); ville des intelligences plutôt que ville intelligente, selon la formule reprise par de nombreux édiles, depuis Anne Hidalgo à Paris et Jean Rottner à Mulhouse ; citoyen capteur plutôt que consommateur capté, selon l'heureux slogan de la Fing (Fédération Internet Nouvelle Génération); wiki city contributive plutôt que néocybernétique totalitaire. Le numérique au service du citoyen, de son ingéniosité et de son agilité.

Il ne suffit néanmoins pas d'affirmer le primat de l'humain sur la technique pour régler le dossier ! Les agglomérations européennes 
semblent surtout, aujourd'hui encore, manier le marketing territorial, à coup de mesures ponctuelles. Les enjeux n'en sont pas moins réels. Ils commandent de faire le tri entre permanences et changements.

La ville intelligente poursuit une double tradition : celles des réseaux techniques du XIX ${ }^{\mathrm{e}}$ siècle, celle des bases de données urbaines de la deuxième moitié du $\mathrm{XX}^{\mathrm{e}}$ siècle. Dans ces domaines, les continuités l'emportent sur les ruptures. En revanche, le développement du numérique dans les systèmes urbains offre la possibilité d'un utile changement de regard sur les villes, dans leurs mutations métropolitaines : elle permet une intelligibilité des usages. Elle fournit matière à une explicitation des contours de la ville évènementielle, de la ville servicielle, de la ville des flux et des circulations, loin de la doxa urbanistique focalisée sur la morphologie des villes.

Une première partie met en évidence la capacité des réseaux techniques et numériques à créer de nouveaux services ou à améliorer les services existants, en démultipliant les potentialités de transactions entre acteurs individuels ou collectifs, publics ou privés. Une deuxième partie relativise, en revanche, l'aptitude des datas à encourager une action publique transversale, débarrassée des logiques de silo. La troisième partie se concentre alors sur l'apport le plus significatif de la smart city : une meilleure connaissance des usages de la ville, devenue territoire des flux et des circulations, des rythmes et des évènements. La quatrième partie souligne la place privilégiée du smartphone dans ces vies mobiles, outil et symbole du changement de paradigme urbanistique. La cinquième partie plaide pour rendre ces mutations visibles et intelligibles, dans l'espace public, à travers des infographies à taille urbaine, mettant en scène les métabolismes de la métropole contemporaine.

\section{Réseaux techniques : optimiser pour faire « marché "}

La mise en réseau des territoires ne date ni d'Internet ni de l'automobile. On fixera volontiers la naissance des macro-systèmes techniques territoriaux à l'invention par les frères Chappe du sémaphore optique, qui va révolutionner les communications, publiques et privées, en France à partir de la fin du XVIII ${ }^{\mathrm{e}}$ siècle. L'assainissement, la distribution d'eau, le gaz et l'électricité, le tramway, fabriqueront au $\mathrm{XIX}^{\mathrm{e}}$ siècle un urbanisme des réseaux, qui trouvera son épanouissement au $\mathrm{XX}^{\mathrm{e}}$ siècle avec le développement de la voiture individuelle.

Quelques spécificités nationales - fragmentation communale, grands corps de l'État - vont produire un modèle français des services en réseaux : de grandes entreprises publiques monopolistiques pour les réseaux nationaux (électricité, chemin de fer, poste, télécommunication), de grands groupes privés gérant sous forme de délégation de service ou de concession dans les réseaux locaux (eau, assainissement, chauffage urbain, transport public).

Une démarche qualifiée de néo-libérale, introduite dès les années 1970 aux États-Unis et relayée par l'Union européenne à partir du milieu des années 1980, va enclencher un double mouvement de privatisation et de dérégulation 
(fin des monopoles). Ce rappel historique succinct permet de comprendre l'état actuel du capitalisme urbain en France : acteurs traditionnels en quête de nouveaux marchés (les majors de l'eau et des transports urbains, les énergéticiens « recomposés », la Poste et la SNCF), entreprises d'ingénierie (tels Thalès ou Dassault Systèmes) ou du BTP diversifiant leurs activités, nouveaux entrants comme les grandes entreprises de l'informatique et des télécommunications (IBM, Cisco, Orange) voyant dans l'urbain un domaine de déploiement prometteur pour leur business, développeurs et start-ups de tout poil, en particulier autour de la « mobilité intelligente » et des promesses de l'économie collaborative... La concurrence apparaît rude, d'autant qu'il en existe encore beaucoup d'autres cherchant à occuper une place stratégique dans la chaîne de valeur de la ville intelligente, grandes enseignes de bricolage (intéressés par le devenir de l'habitat), électriciens (promoteurs de la domotique) ou vendeurs de mobilier urbain (connectant l'espace public).

Le mot d'ordre est l'optimisation. Mieux réguler par une meilleure information. S'il y a cent ans que l'on régule le trafic automobile, on comprend bien que capteurs en temps réel et traitement de données peuvent permettre quelques progrès. Réduction des fuites dans les canalisations de distribution d'eau potable, intensité de l'éclairage nocturne en fonction des présences humaines, tarification du ramassage des ordures ménagères selon le poids des poubelles...

Ces objectifs ne vont pas de soi, puisqu'ils inversent le modèle économique traditionnel des services en réseau, dont les entreprises gagnent d'autant plus d'argent qu'elles manipulent des volumes importants. Ces démarches cherchent au contraire à réduire les consommations, à raisonner l'usage des ressources. Et, lorsque le jeu des acteurs et les relations contractuelles s'y prêtent, elles favorisent aussi une montée en compétence des collectivités territoriales dans les secteurs afférents. Beaucoup de ces innovations, en effet, supposent un intérêt pour la demande de services, les usages, et pas seulement l'offre fournie par le prestataire.

Les « nouveaux entrants » apportent par ailleurs leurs capacités d'innovation dans un champ technico-politique, l'urbanisme, marqué par un fort conservatisme des doctrines et des instruments. C'est particulièrement le cas pour les politiques de déplacement, qui commencent à être sérieusement bousculées par de nouveaux modèles technico-économiques façon Blablacar ou Vulog (covoiturage organisé et technologies d'autopartage).

L'intelligence de l'affaire tient, en l'occurrence, dans une adéquation ajustée entre l'offre et la demande de mobilité, par plus d'information et plus de confiance. Élargi et optimisé, le marché peut ainsi trouver une rentabilité (ou un moindre recours à l'argent du contribuable) inatteignable précédemment, à l'instar du transport public dans les territoires périurbains. Les évolutions en matière énergétique sont du même ordre, mutatis mutandis, en facilitant des régulations plus locales avec des offres plus diversifiées (l'habitant devenant ainsi producteur, avec ses panneaux solaires, avec ses batteries électriques, avec des énergies renouvelables intermittentes). Les smart grids concrétisent la libéralisation du marché de l'énergie, auparavant piloté de manière 
centralisée par un acteur monopolistique.

In fine, l'intelligence des réseaux semble autant profiter au " système » qu'à l'usager ; un peu comme dans le chemin de fer : avant, les trains arrivaient à l'heure ; aujourd'hui, moult applications, y compris collaboratives, permettent au voyageur de savoir comment anticiper, gérer, pallier le retard de son train ! Faute d'une certitude de l'offre, on aide la demande à s'adapter. Vive le progrès ?

Ainsi, les belles ambitions de sobriété environnementale s'inscrivent dans un moment politico-économique de l'évolution des services en réseau qui fait de la quête d'optimisation un outil de fluidification des marchés autant que de développement durable. En tout état de cause, la continuité l'emporte sur la rupture, l'« intelligence » des réseaux facilitant une mise en transaction généralisée des industries de service. Qu'en est-il en revanche lorsqu'est promue l'idée d'interface ou d'interrelation entre services ? Cette vision de la ville comme système de systèmes n'est pas neuve. Elle se nourrit des métaphores organicistes, chères à la pensée écologiste, comme des modèles techniques promus par les cabinets d'ingénierie. Elle rejoint une injonction récurrente dans l'action publique : décloisonner !

\section{Datas et gouvernance : les velléités d'une cybernétique apolitique}

Bases de données urbaines, systèmes d'information... La relecture de la littérature des années 1970 sur ce que l'on appelait alors, avec le rapport Nora-Minc, l'informatisation de la société est cocasse : mêmes espoirs dans l'intégration automatisée de données provenant de plusieurs sources, mêmes désillusions à l'égard d'outils d'aide à la décision niant les processus sociopolitiques. Quatre décennies plus tard, les analyses de l'ouvrage de Pierre Grémion et Haroun Jamous sur L'ordinateur au pouvoir restent pertinentes.

Ainsi le site Internet d'IBM nous apprend-il que «IBM Intelligent Operations Center aide les responsables des pouvoirs publics à gérer des environnements, des incidents et des urgences complexes pour les municipalités grâce à une solution conçue pour les villes qui fournit des éclairages opérationnels. Elle propose une visualisation des données intégrée, une collaboration quasiment en temps réel et des analyses approfondies pour aider les organismes municipaux à améliorer l'efficience continue des opérations municipales, à planifier la croissance et à gérer la réactivité ».

Le glissement est tentant, entre le « centre de contrôle » des flux de la ville et le tableau de bord de l'action publique. Mettre en commun des données autoriserait à dépasser les fameuses logiques de silo. Penser «mobilité » en regroupant les données des divers transports collectifs, du trafic automobile, de la marche et du vélo, etc. Penser « environnement » en intégrant les indicateurs des producteurs de données aux échelles urbaines, départementales et régionales. La data, et mieux encore l'open data, serait apte à franchir les frontières organisationnelles (au sein des services d'une même structure) ou institutionnelles.

Rien néanmoins ne permet aujourd'hui de soutenir que la mise en circulation de données construit 
ou facilite l'élaboration de ce que les politistes appelleraient une gouvernance intersectorielle et multiscalaire. La théorie comme la pratique désignent en revanche de vraies explications aux lacunes dans la transversalité et la coordination des politiques publiques locales : faible culture du management par projet dans les services administratifs, forte division du travail politique par la multiplication des délégations d'élus (au niveau municipal) et des vice-présidences (au niveau des intercommunalités), sectorisation des politiques nationales, ciblage des interventions locales... L'innovation technologique peut éventuellement aider l'innovation institutionnelle. Elle sert parfois à révéler les difficultés organisationnelles, chahuter des rapports de force. Mais elle ne saurait d'aucune façon remplacer les nécessaires évolutions de la culture technico-politique locale.

À l'instar de Saskia Sassen, certains dénoncent aussi la perte de résilience provoquée par une intégration trop poussée entre systèmes urbains, qui figerait les pratiques, les techniques et les acteurs dans un état dont la stabilité serait génératrice d'obsolescence. Le planificateur urbain (intelligent!) se méfie également des injonctions à la cohérence, à l'harmonie, qui nient le caractère dynamique des évolutions territoriales. Et le politiste préfèrera encourager à la coordination entre acteurs, à l'alignement et à la convergence des actions.

\section{Usages : les espaces-temps en partage}

Optimisation marchande des flux de la ville ; espoirs naïfs (ou hypocrites) en une désectorisation des interventions publiques... L'apport de la smart city pourrait s'avérer à la fois plus modeste et plus essentiel : une meilleure connaissance de la ville en marche. Nous appréhendons très mal les fonctionnements urbains. Les urbanistes disposent surtout de statistiques de stocks : nombre d'habitants, d'emplois, de $\mathrm{m}^{2}$ de bureaux, de voitures ; longueur des pistes cyclables, surface des jardins... Des données de notaire ! L'expertise des usages est lacunaire, même si chacun s'emploie à combler cette carence cognitive en faisant appel à ses propres expériences (au point parfois d'en faire une vérité générale...).

Sauf à disposer de travaux académiques $a d$ hoc ou d'enquêtes spécifiques aussi coûteuses que rares, la connaissance et la compréhension des pratiques de la ville restent un trou noir. Cette situation est d'autant plus préoccupante à l'ère des métropoles, parcourues non seulement par leurs habitants mais aussi par tous ceux, en proportion croissante, qui y passent : étudiants, travailleurs, consommateurs, touristes et autres nomades... On sait calculer des densités mais pas des intensités, des localisations mais pas des fréquentations. Or, comment concevoir et exploiter une voirie, un espace public, sans cela ? Comment programmer des développements commerciaux et des équipements publics ? L'urbanisme est trop encore affaire d'offre.

Des penseurs de la ville suggèrent depuis longtemps, à l'instar de Thierry Paquot, de mettre en œuvre un chrono-aménagement ; autrement dit d'intégrer dans le travail de l'urbaniste la quatrième dimension de l'espace : le temps. Il s'agit d'analyser les pulsations urbaines, les rythmes auxquels les bâtiments et les espaces se remplissent et se vident ; les temporalités du travail, 
dans un moment de mutations professionnelles conséquentes ; les horaires et temps de fréquentation des services collectifs; les arbitrages entre activités à l'intérieur et à l'extérieur du logis, etc. Ces informations inédites, dont la smart city rend le recueil simple, ouvrent des champs d'intervention tout aussi inédits à l'action publique : la réduction des pointes de trafic en est l'exemple le plus parlant mais il y a aussi la mutualisation des équipements, l'agencement et la praticité de la voirie au regard des diversités d'usages dans l'espace et dans le temps, les horaires des services, les nouveaux modes d'appropriation des espaces publics... et une fois encore tout ce qui a trait à la «mobilité intelligente».

Les politiques de déplacement buttent sur une double impasse méthodologique. Elles s'intéressent à l'offre de transport (les infrastructures et les matériels qui les utilisent) et pas à la demande de mobilité (pratiques « spatio-temporelles» concrétisant des programmes d'activités); or c'est aussi en régulant cette demande que l'on peut agir sur le système de déplacement. Elles voient le monde des transports de manière monomodale (interventions sectorisées par type de moyen de transport) alors que l'habitant comme l'usager raisonnent en chaînes «multimodales » de déplacement. Le numérique permet une meilleure connaissance des usages et sait réunir à travers des applications mobiles l'ensemble du « marché » des déplacements.

Cette expertise des usages devrait aussi pouvoir s'appuyer - sous réserve d'évaluation des premiers emplois des civic-tech - sur les nouvelles formes de concertation permises par Internet et le numérique, entre productions de données collaboratives et co-constructions citoyennes (toutes expressions qui demanderaient moult développements...).

Quelle qu'en soit la manière, produire de l'information sur les usages présente un autre intérêt majeur, indirect. Cela modifie la représentation dominante de la ville, la maquette et ses avatars ; relativise les questions de forme (la ville des architectes, à le dire rapidement) pour donner toute leur place aux aspects de fonctionnement : le métabolisme plus que la morphologie. Il est aussi permis de prendre ainsi acte de la diversification croissante des pratiques : ville à la carte, zapping territorial, face à la vision d'un passé plus normé dans ses catégories socio-spatiales. L'intelligence des usages se saisit de la ville éphémère, que les statistiques font disparaître derrière les moyennes. Elle fournit matière à une explicitation des contours de la ville évènementielle, au-delà des routines quotidiennes de la vie urbaine.

L'approche « chronotopique » fait de l'espace urbain une sorte de palimpseste mouvant, qui suppose des qualités encore peu courantes d'adaptabilité et de réversibilité. Le marché devant l'église ou le cirque sur la grande place illustrent l'ancienneté de l'éphémère urbain. Mais le processus de métropolisation - comme «mise en réseau » des territoires - accélère cette mise en mouvement de la ville. Surtout, congruence bienvenue, le chrono-urbanisme trouve son instrument d'analyse dans les informations potentiellement produites par la smart city. Il y a donc matière à une action publique urbaine renouvelée, qui prenne au sérieux ces questions, qui sache inventer des managers des temps, moins maitres des horloges que « chorégraphes urbains » (selon 
l'expression de Luc Gwiazdzinski) en quête des bons tempos à faire partager.

Les bureaux ou maison des temps, sporadiquement réclamés mais rarement mis en place, trouveraient sans doute plus de crédibilité et de ressources en s'appuyant sur ces données originales, susceptibles de fournir des marges de manœuvre jusqu'alors peu perçues ou mal comprises en termes de régulations temporelles. Ces institutions ou dispositifs seront légitimes s'il existe du « grain à moudre » pour les « producteurs de temporalités » qu'il convient de faire asseoir autour d'une même table : monde de l'enseignement, entreprises, hôpitaux, grandes administrations publiques, commerces...

Cette « mise en musique » des agendas doit aussi compter avec des citadins acteurs, qui s'adaptent en temps réel à leur situation. L'exemple paradigmatique est fourni par Waze, application de trafic et de navigation, où chacun informe la « communauté » de l'état du trafic. Comme l'écrit le site Internet de Waze, rien ne peut battre de véritables personnes : «Imaginez des millions de conducteurs sur les routes, travaillant ensemble avec un objectif commun : déjouer le trafic». Quitte à passer devant des écoles ou à travers des quartiers jusqu'alors calmes... ce qui souligne la nécessité d'une régulation publique pour traiter ce genre de sujet, encore à construire.

Antoine Picon, l'un des premiers aux États-Unis et en France à avoir pensé la ville intelligente, $\mathrm{y}$ voit la promesse d'" une pensée des situations et des scénarios urbains », à travers la notion de ville évènement. C'est aussi une façon de dire qu'à côté des vieilles logiques planificatrices, qui tentent sinon de maîtriser du moins de fournir des règles ou un cadre du développement urbain à moyen terme, une ville du temps présent mérite toute l'attention de l'urbaniste et de l'élu local.

\section{Géolocalisation : l'insensé retour du hic et nunc}

Le smartphone est devenu en quelques années le couteau suisse du citadin métropolitain. Il sauve du chaos bien des grandes villes africaines, il sauve de l'enfermement bien des adolescents français ! La convergence des deux innovations majeures qui font le smartphone, le téléphone mobile et le GPS, est d'ailleurs peut-être un hasard de l'histoire... Que se serait-il passé si une suite répétée d'échecs de lancement avait bouleversé les plans de l'armée américaine dans son ambition de déployer les satellites du système Navstar? (les déboires du système de téléphonie mobile par satellites Iridium ou le retard pris par Galileo, le système européen concurrent du GPS américain, laissent entrevoir que l'hypothèse est crédible). Que se serait-il passé si la guerre du Golfe n'avait pas eu lieu et si l'armée américaine avait alors continué à dégrader le signal du Global Positioning System (30 mètres de précision) ? Manquant de GPS militaires, elle a dû retirer la distorsion du système afin d'utiliser des GPS civils ; et elle ne l'a pas rétablie.

Nous aurions disposé de téléphones mobiles qui nous auraient permis de faire mille choses, mais pas de transformer la ville en espace de jeux (Ingress, Pokémon Go...), pour conquérir des territoires ou retrouver des indices virtuels (geocaching), pas de draguer avec des « profils » 
se trouvant à proximité (application Tinder), pas d'arrêter de se perdre lorsqu'on ne maîtrise pas l'orientation cartographique, pas de connaître les services de voisinage. Le mobilier urbain devient un relais privilégié pour fabriquer un citadin «massivement » connecté. Le risque de transformer cet espace public « augmenté » en grand équipement commercial n'est pas nul. Mais ces projets peuvent aussi contribuer à faire découvrir les plaisirs de la ville, de la proximité et de la densité à des usagers jusqu'alors éloignés de cette culture de l'urbanité.

Le smartphone active ainsi des territorialités faites de liens autant que de lieux. La géolocalisation associe le proche et le lointain, la contiguïté et la connexité. Processus de mise en réseau des territoires, la métropolisation trouve son outil du quotidien des vies mobiles. «T'es où ? », se demandent désormais les « urbains généralisés ».

Une fois encore, l'abolition des distances déjà promise depuis le chemin de fer (qui va si vite au XIX ${ }^{\mathrm{e}}$ siècle, à lire les auteurs de l'époque), la fin des villes déjà annoncée par la fée électricité (pourquoi s'amonceler près des sources d'énergie dès lors que l'on sait la transporter ?) ne se concrétisent pas. L'omniprésence téléphonique et télévisuelle, qui devait renvoyer spectacles vivants et rendez-vous en face-à-face à la préhistoire ubiquitaire, n'entame pas la valorisation du de visu. Il y a toujours une ruse de l'histoire pour revitaliser l'urbain, compris comme dispositif de maximisation des interactions sociales : l'économie devient relationnelle, la mobilité s'avère dispendieuse. Et l'outil hybride GPStéléphone-Internet réenchante nos rues !

\section{Métabolisme urbain : des flux à rendre visibles}

La smart city peut donc aider les urbanistes à sortir du malaise que Gabriel Dupuy détecte dans les méthodes et dans les doctrines de l'aménagement, issues d'un modèle de développement urbain obsolète, initié après la Seconde Guerre mondiale. C'est en tout cas une aide précieuse pour les tenants d'un urbanisme des usages, plus attentif aux pratiques qu'aux formes, aux dynamiques qu'aux stabilités.

La smart city présente un autre intérêt potentiel majeur : rendre sensible l'importance de la technique dans l'aménagement et le développement des villes. Ce que les macro-systèmes techniques urbains, protégés par l'opacité des boîtes noires et des réseaux, n'avait pas rendu possible est paradoxalement encouragé par l'évidence technologique de la smart city : un questionnement sur le formatage de la ville par les outils, impensé récurrent de la fabrique urbaine. Cette ouverture démocratique suppose une prise en charge publique de la question. Des collectivités s'y emploient, qui cherchent à inventer des formes inédites de relation public-privé et de gouvernance pour reprendre la main. La prise de conscience serait aussi facilitée par la mise en évidence concrète des flux du métabolisme urbain.

Alors que les préoccupations environnementales commandent de s'intéresser chaque jour un peu plus à l'air, à l'eau, à l'énergie, aux circulations de toutes sortes, notre perception de l'espace reste - on l'a déjà évoqué - focalisée sur le bâti, la morphologie; l'immobile plus que le mobile. Certes, quelques enseignes de pharmacie affi- 
chent la température ambiante. Certes, quelques panneaux à messages variables autoroutiers informent d'un pic de pollution. Mais le tableau de bord des fonctionnements métropolitains, en filigrane derrière les écrans d'ordinateur des collectivités territoriales et des opérateurs de réseaux, n'a pas encore trouvé sa place publique. Bleus lorsque la marée monte, verts quand elle descend, les pylônes bordelais du Pont Chaban sur la Garonne participent, par leurs superbes lumières nocturnes, de cet objectif de mise en scène des flux, en l'occurrence des courants. Depuis l'été 2016, Montréal a poussé plus loin le concept : le pont Jacques Cartier s'illumine toutes les nuits, au rythme des saisons, des jours et des heures ; selon des données sur la météo, la circulation, l'actualité. La mention de Montréal sur les réseaux sociaux fait varier l'intensité et la vitesse des scintillements lumineux. Connexions vivantes est ainsi le «baromètre de la vie dans la métropole et de l'énergie des Montréalais».

Ce compteur urbain XXL a des prédécesseurs. À l'initiative d'énergéticiens soucieux de faciliter des comportements plus sobres, des designers ont élaboré des horloges énergétiques, par exemple pour indiquer en temps réel la consommation d'un bâtiment. Immatérielle, la consommation électrique devient visible. Changer d'échelle pour donner à voir de manière attractive et pédagogique les consommations (et productions) énergétiques d'une métropole ne pose aucun problème technique rédhibitoire. Il en serait de même pour l'eau, les déchets, le niveau sonore, la qualité de l'air... Des plasticiens, concernés par le développement durable, imaginent pour leur part de nouvelles esthétiques créant les conditions d'une expérience sensorielle de la ville des flux : une corde suspendue, une image sur grand écran, une sphère lumineuse, une fontaine... dont les mouvements, apparences, intensités se corrèlent à divers paramètres urbains.

Datas, Internet des objets, connectivités humaines généralisées... Le nouveau monde numérique offre déjà mille possibilités de jouer avec des infographies urbaines, à la taille d'un pont, d'un mur ou d'un banc. Les projets qui se multiplient sous l'étendard de la « ville intelligente » gagneront à intégrer cette ambition citoyenne : une visibilité, directe ou médiatisée, des métabolismes urbains, gage de leur intelligibilité pour tous les usagers et habitants, gage de mobilisation des acteurs locaux pour des politiques publiques attentives aux transitions sociétales.

Les changements de paradigme ont besoin de marqueurs et de symboles. Ces infographies à taille urbaine joueront ce rôle à merveille, pour signifier l'avènement d'un urbanisme où la pierre sait accueillir la chair (pour paraphraser le titre du livre fameux de Richard Sennet). Ainsi s'esquisse la métropole contemporaine, dans un enrichissement lexical majeur : le hard, l'objet, la forme laissent place au soft, à l'usage, au métabolisme. L'articulation entre stocks et flux, bâtis et circulations, sédentarité et mouvement, devient ou redevient l'enjeu majeur des gouvernances métropolitaines à inventer. 


\section{$R \cdot E \cdot F \cdot E \cdot R \cdot E \cdot N \cdot C \cdot E \cdot S \quad R \cdot E ́ E \cdot S \cdot U \cdot M \cdot E ́$}

COURMONT Antoine, « Open data et recomposition du gouvernement urbain : de la donnée comme instrument à la donnée comme enjeu politique ", in Informations sociales, $\mathrm{n}^{\circ} 191$, Paris, 2015/5, p. 122-135.

DUPUY Gabriel, "L'avenir de la smart city », in Urbanisme, $\mathrm{n}^{\circ}$ 394, Paris, automne 2014, p.34-35.

GRÉMION Pierre et JAMOUS Haroun, L'ordinateur au pouvoir. Essai sur les projets de rationalisation du gouvernement et des hommes, Seuil, Paris, 1978.

GWIAZDZINSKI Luc, « Face aux nouveaux régimes temporels métropolitains, les pistes du chrono-urbanisme pour une ville malléable », in Urbia, $\mathrm{n}^{\circ} 16$, université de Lausanne, février 2014, p. 179-195.

MAUGARD Alain, « La smart city, ou comment vivre avec des modes de vie durables en ville », in Egis Contact, $\mathrm{n}^{\circ}$ 42, Paris, décembre 2016, p. 3-4. PICON Antoine, Smart Cities A Spatialised Intelligence, Wiley, Chichester, 2015.

PISANI Francis, Voyage dans les villes intelligentes : entre datapolis et participolis, Netexplo, Paris, 2015.

SENNETT Richard, La Chair et la pierre, éditions de la Passion, Paris, 2002.
Au-delà des légitimes critiques adressées à la rhétorique de la smart city, le développement du numérique dans les systèmes urbains peut contribuer à un utile changement de regard sur les villes, dans leurs mutations métropolitaines.

Nouveaux services individuels ou collectifs, meilleure connaissance des pratiques et des rythmes urbains, la smart city permet une intelligence des usages. Elle fournit matière à une explicitation des contours de la ville évènementielle, de la ville servicielle, loin de la doxa urbanistique focalisée sur la morphologie des villes.

Couteau suisse du citadin métropolitain, le smartphone active des territorialités faites de liens autant que de lieux. La géolocalisation associe le proche et le lointain, la contiguité et la connexité. Processus de mise en réseau des territoires, la métropolisation trouve ainsi son outil du quotidien des vies mobiles. Les espaces publics y trouvent de nouvelles vocations.

Portée tant par les traditionnels gestionnaires des services en réseau que par de grandes et petites entreprises du numérique, la smart city rend visible l'importance de la technique dans l'aménagement et le développement des villes. Ce que les macrosystèmes techniques urbains, protégés par l'opacité des boîtes noires et des réseaux, n'avaient pas rendu possible est paradoxalement encouragé par l'évidence technologique de la smart city. Des infographies urbaines restent à imaginer, qui rendent visibles les métabolismes urbains.

Ainsi s'esquisse la métropole contemporaine, dans 
un enrichissement lexical majeur : le hard, l'objet, la forme laissent place au soft, à l'usage, au métabolisme. L'articulation entre stocks et flux, bâtis et circulations, sédentarités et mouvements, devient ou redevient l'enjeu majeur des gouvernances métropolitaines à inventer.

\begin{abstract}
While we can legitimately criticise the rhetoric swirling around the smart city, increased use of digital technology in urban systems really can lead to positive changes in how we look at major cities and population centres.
\end{abstract}

Smart cities can provide intelligent new individual and collective services, and provide a better understanding of how, when and why we use our cities. They allow us to view and understand the city as a space for events and services, a step away from traditional town planning focused on urban morphology.

The smartphone has become a tool for the modern citydweller, a Swiss-army knife which combines physical places and virtual links to carve out new neighbourhoods. Thanks to GPS, the line between near and far, friend and neighbour, has blurred. Smartphones are now becoming a core tool to connect districts, neighbourhoods and people, offering a new lease of life to existing public spaces.

Riding on the backs of traditional network service providers and digital service companies of all sizes, smart cities are casting light on the importance of technology in urban planning and development. Where technical urban macro-systems failed, hidden by the cloak of black boxes and networks, smart cities are, paradoxically, succeeding. Now all we need is a clear graphical representation of our urban spaces to show us exactly how they live and breathe.

This is the future of the modern metropolis, where the changes are felt deep in the very language we use: the hard, the object and form, is giving way to the soft, the uses and the city's metabolism. The connections between stocks and flows, buildings and traffic, stillness and movement are becoming (some not for the first time) the new key issues for metropolitan authorities to address. 
